\title{
Star Formation in Relic HII Regions of the First Stars: Binarity and Outflow Driving
}

\author{
Masahiro N. Machida ${ }^{1,2}$, Kazuyuki Omukai ${ }^{1}$, and Tomoaki Matsumoto ${ }^{3}$
}

\begin{abstract}
Star formation in relic HII regions of the first stars is investigated using magneto-hydrodynamical simulations with a nested grid method that covers $\sim 10$ orders of magnitude in spatial scale and $\sim 20$ orders of magnitude in density contrast. Due to larger fraction of $\mathrm{H}_{2}$ and $\mathrm{HD}$ molecules, its prestellar thermal evolution is considerably different from that in the first star formation. Reflecting the difference, two hydrostatic cores appear in a nested manner: a protostar is enclosed by a transient hydrostatic core, which appears during the prestellar collapse. If the initial natal core rotates fast at a rate with rotational to gravitational energy ratio $\beta_{0} \gtrsim 0.01-0.1$, the transient hydrostatic core fragments to $\sim 10 M_{\odot}$ sub-cores at density $\sim 10^{9} \mathrm{~cm}^{-3}$. With smaller rotation energy, fragmentation occurs at higher density while a single protostar forms without fragmentation if rotation is extremely slow with $\beta_{0} \lesssim 10^{-6}-10^{-5}$. If magnetic field is present, these threshold values of $\beta_{0}$ is boosted owing to angular momentum transport by the magnetic breaking. Magnetic field also drives the protostellar outflows. With strong magnetic field, two distinct outflows are observed: The slower one emanates from the transient hydrostatic core, while the faster one from the protostar. These flows may affect the final stellar mass by ejecting some of masses in the initial core, and also may play some role in driving and maintenance of interstellar turbulence in young galaxies.
\end{abstract}

Subject headings: cosmology: theory — galaxies: formation — stars: formation

\section{Introduction}

Theoretical studies in the last decade revealed that first stars in the universe were born in redshift $z \sim 20-30$ as very massive stars with $\gtrsim 100 M_{\odot}$ (e.g., Ciardi \& Ferrara 2005).

\footnotetext{
${ }^{1}$ National Astronomical Observatory of Japan, Mitaka, Tokyo 181-8588, Japan; omukai@th.nao.ac.jp

${ }^{2}$ Department of Physics, Kyoto University, Kyoto 606-8502, Japan; machiam@scphys.kyoto-u.ac.jp

${ }^{3}$ Faculty of Humanity and Environment, Hosei University, Chiyoda, Tokyo 102-8160
} 
It is also suggested that a fraction of them are formed as binaries or multiples as long as their natal cores somewhat rotate (Machida et al. 2008a; Turk, Abel, \& O'Shea 2009). To drive protostellar outflows, ubiquitously observed in the solar neighborhood and affecting the protostellar accretion, from the first stars, however, requires a magnetic field of $10^{-8}-10^{-9} \mathrm{G}$ at $10^{3} \mathrm{~cm}^{-3}$ (Machida et al. 2006a), higher than the level generated by mechanisms currently proposed, e.g., Biermann batteries at the cosmic recombination or halo virialization (Ichiki et al.; Xu et al. 2008) by 2-3 orders of magnitudes. Hence the outflows/jets are hard to emerge at least in the early phase of protostellar accretion.

Even in the primordial gas, prestellar thermal evolution, which affects fragmentation and thus characteristic stellar mass, can differ in a cloud disturbed by a former generation of stars. The stars born in such an environment begins to be recognized as a different class of objects and called Population III.2 stars, while the genuine first stars are Population III.1 stars (O'Shea et al. 2008). Various feedbacks can affects cradles of subsequent generation of stars, and thus Pop III.2 in principle includes distinct sub-classes. The most studied example, though, is those formed in relic HII regions of the first stars.

A large HII region forms around a massive first star. Pop III stars with mass $\gtrsim 100 M_{\odot}$ end their lives as supernovae only in a limited mass range $\left(140-260 M_{\odot}\right.$; Heger \& Woosley 2002; Umeda \& Nomoto 2002), and thus the most of them would collapse directly as black holes rather quiescently. The surrounding gas is left ionized and starts recombining gradually, and another episode of star formation commences after $\sim 100$ Myrs. In such relic HII regions, abundant electron, which is the catalyst for $\mathrm{H}_{2}$ formation, allows the rapid replenishment of $\mathrm{H}_{2}$ to the fraction $\sim 10^{-3}$, about an order of magnitude higher than in the Pop III.1 case. The cooler environment $(\lesssim 150 \mathrm{~K})$ due to the higher $\mathrm{H}_{2}$ cooling is preferable for HD formation, and its cooling further lowers the temperature down to a few tens of Kelvins, close to the CMB temperature (Nagakura \& Omukai 2005). The fragmentation mass-scale due to the HD cooling is about $\sim 40 M_{\odot}$, and most of it is expected to be accreted by the formed star eventually (Yoshida, Omukai, \& Hernquist 2007; McGreer \& Bryan 2008). Not only in the relic HII regions, but the HD cooling can be important also in a medium ionized at the virialization of a large halo or even in a small halo if dynamical heating during mass assembly is weak (Ripamonti 2007). This "HD mode" of primordial star formation may be a more common mode than the Pop III.1 one in forming galaxies.

In addition, if all the Pop III.1 stars were indeed very massive with a few hundreds of solar mass, they might have been very inefficient cauldrons for metals owing to the limited mass range for supernovae. With smaller mass and being able to explode as Type II supernovae, Pop III.2 stars could be more responsible for the metals in the early universe. To predict its mass range more accurately, we need to know the conversion efficiency from a 
dense core to stars, which is determined by binarity/multiplicity, feedback from protostellar outflow etc. As an approach to this problem, here, we study the prestellar collapse of rotating dense cores that is produced in an initially ionized gas, putting main emphasis on the condition of binary formation and launching of outflows.

This paper is organized as follows. The numerical method is briefly described in $\S 2$, and the results are presented in $§ 3$. Finally, we discuss its significance in the cosmological context and caveats in $\S 4$.

\section{Numerical Method}

First we calculate the temperature evolution by using a free-falling one-zone model of Omukai et al. (2005), starting from number density $1 \mathrm{~cm}^{-3}$, temperature $7000 \mathrm{~K}$ and the initial ionization degree 0.1 . The $\mathrm{CMB}$ radiation at $27.3 \mathrm{~K}$ is imposed. With given temperature evolution as a function of density, we performed magneto-hydrodynamics (MHD) by a nested-grid method to gain high resolution near the center. As the initial condition, we take the critical Bonner-Ebert sphere with central density $10^{4} \mathrm{~cm}^{-3}$ but with a factor 1.68 of density enhancement to induce the gravitational instability. In addition, $m=2$ mode of density perturbations are imposed with $10 \%$ to break axi-symmetry and cause fragmentation. The initial angular momentum and magnetic field are parallel to the z-axis, and are parameterized by the ratio of rotation to gravitational energy, $\beta_{0}$, and the ratio of magnetic to gravitational energy $\gamma_{0}$, respectively. These quantities are related to the initial rotation rate of the core $\Omega_{0}\left(\mathrm{~s}^{-1}\right)$ and magnetic flux density $B_{0}(\mathrm{G})$ as $\beta_{0}=0.1\left[\Omega_{0} / 3 \times 10^{-14}\left(\mathrm{~s}^{-1}\right)\right]^{2}$ and $\gamma_{0}=1.5 \times 10^{-5}\left[B_{0} / 10^{-7}(\mathrm{G})\right]^{2}$, respectively. The magnetic field is assumed to be frozen to the gas. For details of the MHD and initial conditions, see Machida et al. (2005). The initial parameters of calculated runs and summary of the result are presented in Table 1.

\section{Results}

The adopted temperature evolution in the HD mode is shown in Figure 1. Also shown is that in the Pop III.1 case (" $\mathrm{H}_{2}$ mode"), where the collapse begins from an almost neural condition. The temperature in the HD mode is lower than that in the $\mathrm{H}_{2}$ mode for $10^{2-7} \mathrm{~cm}^{-3}$, and reaches several $10 \mathrm{~K}$ owing to the $\mathrm{HD}$ cooling. The HD-cooling rate saturates at $10^{5} \mathrm{~cm}^{-3}$ by collisional de-excitation, and the temperature begins to increase with density. The fragmentation scale $\sim 40 M_{\odot}$ is set around there (Yoshida et al. 2007). At $10^{8} \mathrm{~cm}^{-3}$, the $\mathrm{H}_{2}$ begins to form via the three-body reaction, whose heating makes the temperature jump 
up abruptly. The simultaneous increase of pressure slows down the gravitational collapse near the center of the dense core, and transient hydrostatic core forms just for a brief period. The core begins to collapse dynamically again until the permanent hydrostatic core, i.e., the protostar, forms at $\gtrsim 10^{21} \mathrm{~cm}^{-3}$ unless the centrifugal supports prevents the collapse before that. Similar transient hydrostatic objects, the so-called first cores, form in metal-enriched cores when their central parts become optically thick to the dust absorption.

In metal-enriched cases, the first-core formation is an important epoch for fragmentation of rotating cores (Machida et al. 2009). In the isothermal phase, i.e., before the first-core formation, even though the core becomes flattened by rotation, its collapse does not significantly slow down until the adiabatic temperature-increase leading to the first-core formation. Shortly after the first-core formation, if the core is flat enough, the slow contraction allows perturbations to grow, thereby causing fragmentation into a binary/multiple system. On the other hand, in the $\mathrm{H}_{2}$ mode of primordial star formation (i.e., PopIII.1 formation), where the temperature continues to increase gradually without transient-core formation, no characteristic epoch for binary/multiple formation exists. In this case, when a thin disk forms by rotation, it fragments after further contraction of a few orders of magnitude in density.

The final states of the calculations are presented for different combinations of rotation $\left(\beta_{0}\right)$ and magnetic field strength $\left(\gamma_{0}\right)$ in Figure 2. The cores with initially large rotation $\left(\beta_{0} \gtrsim 0.01-0.1\right.$ in the $\gamma_{0}=0$ cases $)$ fragment shortly after the transient core formation and form wide proto-binaries with initial masses $\sim 1-10 M_{\odot}$ and separations $\sim 10^{4} \mathrm{AU}$ (cases $a$ in Figure 2$)$. Those with smaller rotation $\left(10^{-5} \lesssim \beta_{0} \lesssim 10^{-2}\right.$ in the $\gamma_{0}=0$ cases) fragment at higher density before the protostar formation unless the rotation is extremely small $\left(\beta_{0} \lesssim 10^{-6}-10^{-5}\right.$ in the $\gamma_{0}=0$ cases $)$. Such high-density $\left(10^{17-20} \mathrm{~cm}^{-3}\right)$ fragmentations produce close proto-binaries with initial masses $\sim 10^{-2} M_{\odot}$ and separations $\sim 10^{-2}$ AU (cases $b$ in Figure 2). Note that some models classified in case $b$ show merger of fragments; i.e., models $\left(\beta_{0}, \gamma_{0}\right)=\left(10^{-2}, 1.5 \times 10^{-5}\right)$ and $\left(10^{-4}, 1.5 \times 10^{-5}\right)$. We expect that the merged cores finally evolve in a single massive star, since spiral structures in them effectively transfer the angular momenta outward, which prevents further fragmentation. Those with even smaller rotation collapse all the way without fragmentation and form single protostars (cases $c$ in Fig. 2). While the collapse is approximately spherical, the rotation parameter $\beta$ at the center of the core increases as $\propto n^{1 / 3}$, where $n$ is the number density, from the initial value $\beta_{0}$. When $\beta$ reaches $0.04-0.1$, the radial contraction is retarded by the centrifugal force. The core becomes a thin disk after more contraction by a few orders of magnitude in density (e.g., Machida et al. 2008). The threshold $\beta_{0}$ values (i.e., $0.01-0.1$ and $10^{-6}-10^{-5}$ ) for binary formation correspond to the conditions of thin-disk formation before formation of the transient core $\left(\right.$ at $\sim 10^{8} \mathrm{~cm}^{-3}$ ) and the protostar $\left(\right.$ at $\sim 10^{21} \mathrm{~cm}^{-3}$ ), respectively. 
The magnetic field reduces the angular momentum of the cores through magnetic breaking, hence boosting the threshold rotation rate for fragmentation. For example, with initial magnetic field $\gamma_{0}=1.5 \times 10^{-3}\left(1.5 \times 10^{-1}\right)$, the threshold $\beta_{0}$ for fragmentation increases above $10^{-4}\left(10^{-2}\right.$, respectively) from $\sim 10^{-5}$ in the $\gamma_{0}=0$ case.

Although the initial mass of fragments is very small, they are embedded in a dense core and subsequently grow by accretion until the majority of the core material $\left(\lesssim 10 M_{\odot}\right)$ is accreted by the binary/multiple system (Yoshida et al. 2008). However, if launched, protostellar outflow may reduce the conversion efficiency from the core to the system by evacuating some of envelope materials.

When the initially ordered magnetic field is highly twisted by rotation, outflows are launched either by magnetic centrifugal force or by pinching by magnetic pressure. During dynamical collapse, the cores do not rotate so many times as to twist the magnetic field enough. After hydrostatic core formation, the magnetic field is amplified and distorted owing to the slow contraction, thereby enabling the launching of slow outflows. In the case of present-day star formation, two kinds of outflows appear, corresponding to two epochs

of hydrostatic core formation, i.e., first core and protostar (Machida et al. 2008b). In our case, owing to the presence of the transient hydrostatic core, similar two flows are launched, i.e., the slower one $(2-3 \mathrm{~km} / \mathrm{s})$ at transient core formation, and the faster one $(20-40 \mathrm{~km} / \mathrm{s})$ just at the protostar formation. The speeds of the flows increase with the mass of driving objects, i.e., the transient hydrostatic core and protostar. According to our experiments, the minimum initial magnetic field needed for driving the fast (slow) flow was about $10^{-8} \mathrm{G}$ $\left(10^{-7} \mathrm{G}\right.$, respectively). On the other hand, in the Pop III.1 case, only fast flow is launched as no transient hydrostatic stage exists (Machida et al. 2006a).

\section{Discussion}

Currently, we have no information about rotation rates of Pop III.2 dense cores. If we assume that they are similar to the values in the Milky Way $\left(\beta_{0} \sim 10^{-4}-0.07\right.$ with the median of 0.02; Caselli et al. 2002), fragmentations typically occur at far higher densities than the transient core formation (i.e., case $b$ in Figure 2), and produce close binary/multiple systems. Since the mass scale of the natal cores is $30-40 M_{\odot}$, set by fragmentation induced by the HD cooling (Yoshida et al. 2007), the final product would be a close binary of $10-20 M_{\odot}$ stars.

For the Pop III.1 protostars, only the primordial magnetic field of the cosmological origin is available. On the other hand, the Pop III.2 stars are able to use additional magnetic fields, 
which might have been generated and amplified by activities of previous generations of stars, including both the Pop III.1 and III.2. Therefore, the Pop III.2 protostars could launch the outflow even though an order-of-magnitude larger magnetic field is required for the fast-flow launching than in the Pop III.1 case. To know whether the protostellar jet is launched, we need to wait future numerical simulations to narrow down the possible values for magnetic field in such an environment. If indeed launched, the jet expels some materials in the natal core, and hence reducing the final mass in the binary system. Matzner \& McKee (2000) estimated the efficiency from the core to the system at $25-70 \%$ after the jet feedback in the present-day star formation by way of hydrodynamical simulation. If this efficiency is similar in our case, the final system mass would be $20 M_{\odot}$ out of a $40 M_{\odot}$ core. To know the final mass of stars, we need to follow evolution in the accretion phase, which is left for future studies.

As mentioned in $\S 2$, we used the temperature evolution as a function of density, which is calculated by the one-zone model of Omukai et al. (2005), where spherical clouds with the Jeans lengths are assumed to collapse at about the free-fall rate. In reality, thermal evolution depends on the cloud dynamics, and vice versa. For example, when the cloud shape deviates from the sphere, the optical depths and thus the cooling rates can differ from the assumed values. Also, slower collapse leads to lower compressional heating. Moreover, our method cannot capture the shock heating properly. This could be a significant drawback in simulating more turbulent situations (see Clark et al. 2008 for more discussions).

The first galaxy, harboring the first star clusters, is found to be turbulent owing to the cold accretion flow at its formation by numerical experiments (Wise \& Abel 2007; Greif et al. 2008). Here, however, we have not use turbulent initial conditions for simplicity. If the turbulent energy is comparable to the gravitational one, the cloud evolution can be significantly affected in particular in early phases leading to formation of dense cores (e.g., Clark et al. 2008). Rotational energy of the dense cores would be set by the turbulence and tend to be higher in the more turbulent environment. In this study, we have regarded the degree of rotation as a free parameter and followed the evolution only after the formation of the dense cores.

The protostellar outflows by Pop III.2 stars could contribute to drive and maintain the turbulence in the first galaxies. However, this phase might be short-lived as the first galaxies will be metal-enriched before long. In addition, such metal-free first galaxies might be rare as the previous generations of stars have already polluted with metals. Therefore, protostellar outflows by the Pop II stars would be more important in this context and awaits future studies (e.g., Machida et al. 2009). 
Numerical computation in this work was carried out at the Yukawa Institute Computer Facility. This study is supported in part by the Grants-in-Aid by the Ministry of Education, Science and Culture of Japan (21740136:MM, 19047004, 21684007:KO, 20540238:TM).

\section{REFERENCES}

Caselli, P., Benson, P. J., Myers, P. C., \& Tafalla, M. 2002, ApJ, 572, 238

Ciardi, B., \& Ferrara, A. 2005, Space Science Reviews, 116, 625

Clark, P. C., Glover, S. C. O., \& Klessen, R. S. 2008, ApJ, 672, 757

Greif, T. H., Johnson, J. L., Klessen, R. S. \& Bromm, V. 2008, MNRAS, 387, 1021

Heger, A., \& Woosley, S. E. 2002, ApJ, 567, 532

Ichiki, K., Takahashi, K., Ohno, H., Hanayama, H., \& Sugiyama, N. 2006, Science, 311, 827

Machida, M. N., Matsumoto, T., Tomisaka, K. \& Hanawa, T. 2005, MNRAS, 362, 369

Machida, M. N., Omukai, K., Matsumoto, T., \& Inutsuka, S.-i. 2006a, ApJ, 647, L1

Machida, M. N., Omukai, K., Matsumoto, T., \& Inutsuka, S.-i. 2008a, ApJ, 677, 813

Machida, M. N., Inutsuka, S.-i., \& Matsumoto, T. 2008b, ApJ, 676, 1088

Machida, M. N., Matsumoto, T., \& Inutsuka, S.-i. 2008c, ApJ, 685, 690

Machida, M. N., Omukai, K., Matsumoto, T., \& Inutsuka, S.-i. 2009, MNRAS, submitted

McGreer, I. D. \& Bryan, G. L. 2008, ApJ, 685, 8

Nagakura, T. \& Omukai, K. 2005, MNRAS, 364, 1378

O'Shea, B. W., McKee, C. F., Heger, A., \& Abel, T. 2008, in "First Stars III", xiii, AIP

Ripamonti, E. 2007, MNRAS, 376, 709

Turk, M. J., Abel, T., \& O’Shea, B. 2009, Science, 325, 601

Umeda, H. \& Nomoto, K. 2002, ApJ, 565, 385

Wise, J. H., \& Abel, T. 2007, ApJ, 665, 899

$\mathrm{Xu}$, H. et al. 2008, ApJ, 688, L57 
Yoshida, N., Omukai, K., \& Hernquist, L. 2007, ApJ, 667, L117

This preprint was prepared with the AAS LATEX macros v5.2. 
Table 1: Models and Results

\begin{tabular}{c|cccc|cc}
\hline Model & $\beta_{0}$ & $\gamma_{0}$ & $\Omega_{0}\left[\mathrm{~s}^{-1}\right]$ & $B_{0}[\mathrm{G}]$ & $n_{\mathrm{f}}\left(\mathrm{cm}^{-3}\right)^{a}$ & Outflow $^{b}$ \\
\hline 1 & 0 & 0.15 & 0 & $10^{-5}$ & - & None \\
2 & 0 & $1.5 \times 10^{-3}$ & 0 & $10^{-6}$ & - & None \\
3 & 0 & $1.5 \times 10^{-5}$ & 0 & $10^{-7}$ & - & None \\
\hline 4 & 0.1 & 0.15 & $3.0 \times 10^{-14}$ & $10^{-5}$ & $1.3 \times 10^{18}$ & Both \\
5 & 0.1 & $1.5 \times 10^{-3}$ & $3.0 \times 10^{-14}$ & $10^{-6}$ & $6.7 \times 10^{11}$ & Slow \\
6 & 0.1 & $1.5 \times 10^{-5}$ & $3.0 \times 10^{-14}$ & $10^{-7}$ & $1.5 \times 10^{9}$ & None \\
7 & 0.1 & 0 & $3.0 \times 10^{-14}$ & 0 & $1.5 \times 10^{9}$ & None \\
\hline 8 & $10^{-2}$ & 0.15 & $9.4 \times 10^{-15}$ & $10^{-5}$ & - & Slow \\
9 & $10^{-2}$ & $1.5 \times 10^{-3}$ & $9.4 \times 10^{-15}$ & $10^{-6}$ & $5.4 \times 10^{17}$ & Both \\
10 & $10^{-2}$ & $1.5 \times 10^{-5}$ & $9.4 \times 10^{-15}$ & $10^{-7}$ & $2.4 \times 10^{17}$ & Fast \\
11 & $10^{-2}$ & 0 & $9.4 \times 10^{-15}$ & 0 & $3.7 \times 10^{17}$ & None \\
\hline 12 & $10^{-3}$ & 0.15 & $3.0 \times 10^{-15}$ & $10^{-5}$ & - & Fast \\
13 & $10^{-3}$ & $1.5 \times 10^{-3}$ & $3.0 \times 10^{-15}$ & $10^{-6}$ & $2.1 \times 10^{20}$ & Fast \\
14 & $10^{-3}$ & $1.5 \times 10^{-5}$ & $3.0 \times 10^{-15}$ & $10^{-7}$ & $1.7 \times 10^{20}$ & Fast \\
15 & $10^{-3}$ & 0 & $3.0 \times 10^{-15}$ & 0 & $1.9 \times 10^{20}$ & None \\
\hline 16 & $10^{-4}$ & 0.15 & $9.4 \times 10^{-16}$ & $10^{-5}$ & - & Fast \\
17 & $10^{-4}$ & $1.5 \times 10^{-3}$ & $9.4 \times 10^{-16}$ & $10^{-6}$ & $1.2 \times 10^{22}$ & Fast \\
18 & $10^{-4}$ & $1.5 \times 10^{-5}$ & $9.4 \times 10^{-16}$ & $10^{-7}$ & $2.5 \times 10^{20}$ & Fast \\
19 & $10^{-4}$ & 0 & $9.4 \times 10^{-16}$ & 0 & $2.9 \times 10^{20}$ & None \\
\hline 20 & $10^{-5}$ & 0.15 & $3.0 \times 10^{-15}$ & $10^{-5}$ & - & Fast \\
21 & $10^{-5}$ & $1.5 \times 10^{-3}$ & $3.0 \times 10^{-15}$ & $10^{-6}$ & - & Fast \\
22 & $10^{-5}$ & $1.5 \times 10^{-5}$ & $3.0 \times 10^{-15}$ & $10^{-7}$ & $2.2 \times 10^{21}$ & Fast \\
23 & $10^{-5}$ & 0 & $3.0 \times 10^{-15}$ & 0 & $2.4 \times 10^{21}$ & None \\
\hline 24 & $10^{-6}$ & 0.15 & $9.4 \times 10^{-17}$ & $10^{-5}$ & - & None \\
25 & $10^{-6}$ & $1.5 \times 10^{-3}$ & $9.4 \times 10^{-17}$ & $10^{-6}$ & - & None \\
26 & $10^{-6}$ & $1.5 \times 10^{-5}$ & $9.4 \times 10^{-17}$ & $10^{-7}$ & - & None \\
27 & $10^{-6}$ & 0 & $9.4 \times 10^{-17}$ & 0 & - & None \\
\hline \hline & \multicolumn{7}{c}{} & & & &
\end{tabular}

${ }^{a}$ Number density at fragmentation epoch

${ }^{a}$ Emergence of Slow, Fast and Both (slow and fast) outflows. 


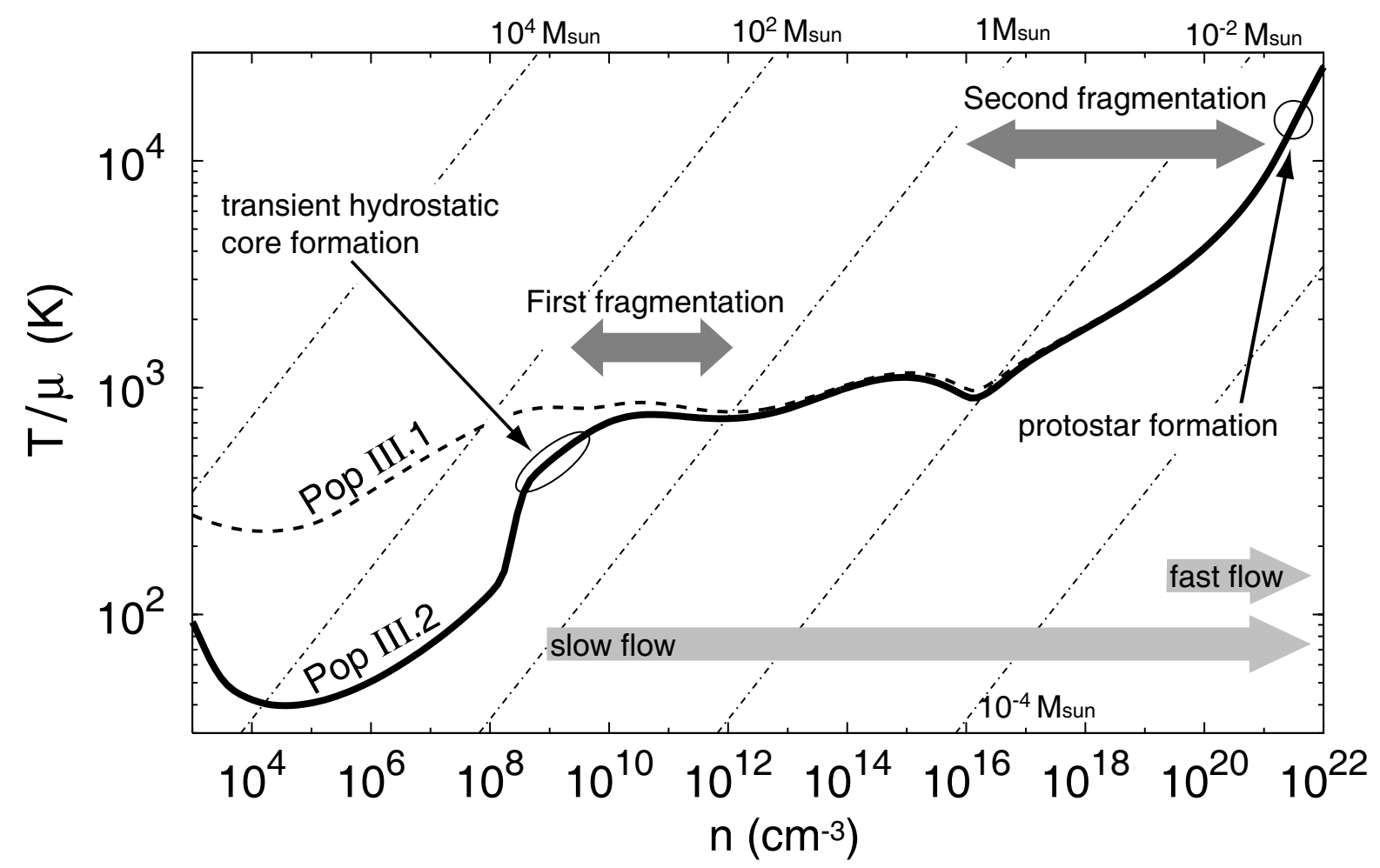

Fig. 1.- Thermal evolution of a metal-free prestellar core from an initially ionized state as function of the number density (thick solid; indicated as "Pop III.2"). The temperature divided by mean molecular weight $\mu$ is shown. Also shown for comparison is that for the Pop III.1 case (dashed). The lines of constant Jeans mass are presented by the thin dot-dashed line. Some characteristic epochs are indicated by arrows with captions. 


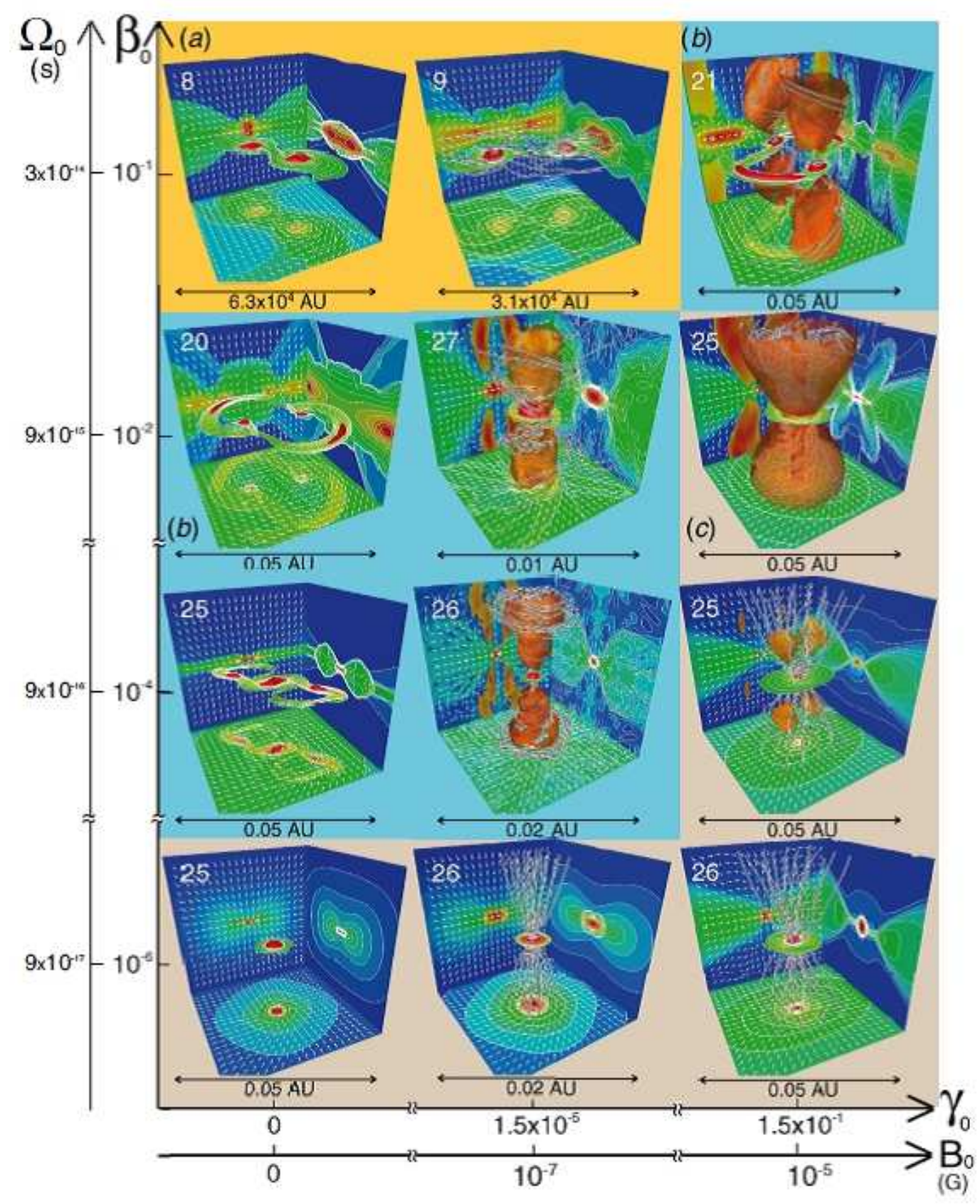

Fig. 2.- The final states for runs with different rotation $\left(\beta_{0}\right)$ and magnetic field strength $\left(\gamma_{0}\right)$. The results are classified according to fragmentation properties; (a) fragmentation shortly after the transient core formation, (b) fragmentation at higher density, and (c) no fragmentation, which are indicated by the background colors. Note the larger binary separations $\left(\sim 10^{4} \mathrm{AU}\right)$ in the cases $(a)$ than those in $\left(b ; \sim 10^{-2} \mathrm{AU}\right)$. In each panel, the structure of high-density region (red isodensity surface) and magnetic field lines (black and white streamlines), and outflow shape (the region of radial velocity $v_{r}>0$; transparent orange surface) are plotted in three dimensions, while the density contour (color and contour lines) and velocity vectors (arrows) are projected on each wall surface. The grid level $l$ and grid scale are shown at the upper-left corners and bottom, respectively, of each panel. 